\title{
FINITELY GENERATED GROUPS WITH VIRTUALLY FREE AUTOMORPHISM GROUPS
}

\author{
by MARTIN R. PETTET
}

(Received 30th January 1994)

\begin{abstract}
It is shown that the full automorphism group of a finitely generated group $G$ is virtually free if and only if the center $Z(G)$ is finitely generated of torsion-free rank $r$ at most two and, depending on the value of $r$, the central quotient $G / Z(G)$ belongs to one of three precisely defined classes of virtually free groups. Some consequences and special cases are also discussed.
\end{abstract}

1991 Mathematics subject classification: 20F28

\section{Introduction}

The main goal of this paper is to obtain at least a coarse classification of the groups of the title.

The general problem of characterizing those groups whose automorphism groups have a prescribed structure would seem to be a reasonably natural one, but it is not one for which anything close to a theory or comprehensive body of results has yet emerged. In view of the kind of pathology that arises even with abelian groups (see, for example, $[12,4.4 .2])$, the problem in its most general form seems likely to remain intractable. However, as with many problems in group theory, positive results can sometimes be obtained by imposing some sort of finiteness condition on the groups in question. For example, one early result of this type was a theorem of $R$. Baer [2] that a periodic group whose automorphism group is finite is itself finite. In the present note, the "finiteness condition" to be exploited is finite generation and so the results may be regarded as extensions of a theorem of J. Alperin [1]: A finitely generated group whose automorphism group is finite is itself either finite or a finite central extension of an infinite cyclic group.

Probably the most obvious example of a group whose automorphism group is virtually free (but not finite) is the free abelian group of rank two (it being well known that $G L_{2}(\mathbb{Z})$ is virtually free). By adapting Alperin's arguments, it is also not difficult to see that any finite central extension of $\mathbb{Z} \times \mathbb{Z}$ also has such an automorphism group. Perhaps a less familiar and more interesting family of examples is the class of groups which are amalgamated free products of two finite groups [6]. Although we are not aware of a reference, it can also be shown without too much effort that any HNN extension of a finite group has a virtually free automorphism group.

We obtain here a description of the groups of the title along lines similar to those of 
Alperin's result, in terms of the structure of the center and of the central quotient (Theorem 3.3). We also include some observations about groups whose automorphism groups are of a much more restricted structure (virtually cyclic or finite-by-free).

As a characterization, Theorem 3.3 is somewhat unsatisfactory because applying it involves determining whether a certain finite subgroup of the outer automorphism group of a free group has finite centralizer. Perhaps the principal effect of the result is to narrow the focus of attention to the question of which finitely generated and virtually free groups have virtually free automorphism groups. Automorphism groups of finitely generated virtually free groups have recently been shown to be finitely presented and virtually of finite cohomological dimension (see [5], [7] and [9]) and so this question represents a very special case of the broader problem of relating the structure of a virtually free group to the virtual cohomological dimension of its automorphism group (see [3] for another special case).

\section{Preliminaries}

We begin with a formal statement of the theorem of Alperin mentioned in the introduction.

Theorem 2.1. [1] Let $G$ be a finitely generated group. Then $A u t(G)$ is finite if and only if $G$ is a finite central extension of a cyclic group.

As an alternate characterization of the groups which arise in Alperin's theorem, it will be convenient to have the following:

Lemma 2.2. A group $G$ is finite-by-cyclic if and only if it is a finite central extension of a cyclic group.

Proof. If $G$ is finite-by-(infinite cyclic), then it is a split extension and, hence, $G$ contains an infinite cyclic central subgroup of finite index. Suppose now that $G$ is a finite central extension of an infinite cyclic group $C$. If $n=|G: C|$, the power map $x \rightarrow x^{n}$ from $G$ into $C$ is the transfer homomorphism (see, for example, [12, 10.1.3]) and so, if $K$ is its kernel, $G / K$ is infinite cyclic and $K \cong K C / C$ is finite.

Finally, a special case of the following technical result will be needed:

Proposition 2.3. [11, Prop. 3.1] Let $G$ be a finitely generated group with a normal subgroup $U$ which is a direct factor of a subgroup $H$ of finite index in $G$ and whose center $Z(U)$ is finitely generated. Let $A=A u t(G)$ and $C=C_{A}(U) \cap C_{A}(G / U)$. Then

(i) There exists a normal subgroup $N$ of $G$ such that $N \leqslant H, U \cap N=1,|G: U N|$ is finite, and $N_{A}(U) \leqslant N_{A}(U N)$.

(ii) $D=C_{A}(U) \cap C_{A}(G / N)$ faithfully induces on $G / U$ a subgroup of finite index in Aut $(G / U)$. 
(iii) $E=C_{A}(N) \cap C_{A}(G / U)$ faithfully induces on $G / N$ a subgroup of finite index in $\operatorname{Aut}(G / N)$.

(iv) $\left|N_{A}(U): C D E\right|$ is finite.

\section{The main theorem and some special cases}

The statement of the main result of this paper is facilitated by some simple notation:

Definitions. We shall say that a group $G$ belongs to the class $\mathscr{F}_{0}$ if there is an extension $F \rightarrow G \rightarrow Q$, where $F$ is free of finite rank and $Q$ is finite, such that if $\chi$ : $Q \rightarrow O u t(F)$ is the corresponding coupling, then $C_{\text {Out(F) }}(\chi(Q))$ is finite.

The subclass $\mathscr{F}_{1}$ consists of all groups $G$ in $\mathscr{F}_{0}$ for which the commutator quotient $G_{a b}=G / G^{\prime}$ is finite.

$\mathscr{F}_{2}$ denotes the class of all finite groups.

The definition of $\mathscr{F}_{0}$ does not seem to suggest an immediate recipe for constructing interesting examples. The obvious examples are those groups which are finite extensions of cyclic groups. Note that the infinite dihedral group actually belongs to $\mathscr{F}_{1}$ whereas any infinite finite-by-cyclic group does not, so the chain $\mathscr{F}_{2} \subseteq \mathscr{F}_{1} \subseteq \mathscr{F}_{0}$ is strictly increasing. In the language of Bass-Serre, it is a fundamental theorem of Karrass, Pietrowski and Solitar that every finitely generated virtually free group is the fundamental group of a finite graph of finite groups (see, for example, [13]). The elements of $\mathscr{F}_{1}$ are precisely the groups in $\mathscr{F}_{0}$ for which this graph is a tree (and thus, they are the ones which may be built up from free products (with amalgamation) of finite groups) and those in $\mathscr{F}_{2}$ are, of course, the groups for which the graph may be taken to be a single vertex. As we show now, a more concise characterization of the class $\mathscr{F}_{0}$ is that it contains precisely the finitely generated virtually free groups whose automorphism groups are virtually free. For the purposes of gaining more intuitive insight into this class, this fact is probably no more illuminating than the definition, but it will at least enable us to extend somewhat our short list of examples.

Proposition 3.1. If $G$ is a finitely generated and virtually free group, then the following are equivalent:

(i) $G \in \mathscr{F}_{0}$;

(ii) $\operatorname{Aut}(G)$ is virtually free;

(iii) $\operatorname{Out}(G)$ is finite.

Proof. If $G$ is virtually free, then so is $\operatorname{In} n(G) \cong G / Z(G)$ and thus, (iii) implies (ii). If (ii) holds, then because a non-trivial finitely generated normal subgroup of a free group necessarily has finite index [8, I.3.11), $\operatorname{lnn}(G)$ is either finite or of finite index in $\operatorname{Aut}(G)$. But $Z(G)$ must be finite or finite-by-(infinite cyclic) and so, by Theorem 2.1 , if $\operatorname{Inn}(G)$ is finite, then so is $\operatorname{Aut}(G)$. Thus, (ii) implies (iii). The remainder of the proof is concerned with the equivalence of (i) and (ii). 
Let $A=A u t(G)$ where $G$ is finitely generated and virtually free. Let $F$ be a free normal subgroup of finite rank and finite index in $G$ and let $Q=G / F$. For any subgroup $X$ of $G$, let $\hat{X}$ denote the subgroup of $A u t(F)$ induced by conjugation by elements of $X$. If $\chi$ : $Q \rightarrow \operatorname{Out}(F)$ is the coupling corresponding to the obvious extension $F \rightarrow G \rightarrow Q$, then $\chi(Q)=\hat{G} / \hat{F}$. We will show that $A$ is virtually free if and only if $C_{\text {Oun(F) }}(\hat{G} / \hat{F})$ is finite.

Suppose that $C_{\text {out }(F)}(\hat{G} / \hat{F})$ is finite. Since $G$ is finitely generated, it has only finitely many subgroups of index $|Q|$ and so, $\left|A: N_{A}(F)\right|$ is finite. Since $N_{A}(F) / N_{A}(F) \cap C_{A}(Q)$ is isomorphic to a subgroup of $A u t(Q)$, it too is finite. If $F$ is non-cyclic, then $\left.C_{A}(F) \cap C_{A}(Q)\right) \leqslant C_{A}(G / Z(F))=1$ and so $N_{A}(F) \cap C_{A}(Q)$ is isomorphic to a subgroup of $A u t(F)$ and indeed, of $C_{A u t(F)}(\hat{G} / \hat{F})$. Since $F \leqslant G, \hat{F} \leqslant C_{A u t(F)}(\hat{G} / \hat{F})$ and so, $C_{\text {out }(F)}(\hat{G} / \hat{F})=$ $C_{A u t(F)}(\hat{G} / \hat{F}) / \hat{F}$. Therefore, $C_{A u t(F)}(\hat{G} / \hat{F})$ is virtually free, whence the same is true of $N_{A}(F) \cap C_{A}(Q)$ and of $A$. Thus, we are reduced to the situation in which $F$ is cyclic.

In this case, $\left|G: C_{G}(F)\right| \leqslant 2$. Now $A u t\left(C_{G}(F)\right)$ is finite by Theorem 2.1 and so $A / C_{A}\left(C_{G}(F)\right)$ is finite. Thus, we may assume that $\left|G: C_{G}(F)\right|=2$. Since $A / C_{A}(Q)$ is also finite, if $C=C_{A}\left(C_{G}(F)\right) \cap C_{A}(Q)$, then $A / C$ is finite. But $C \cong \operatorname{Der}\left(G / C_{G}(F), F\right) \cong \operatorname{Der}(A u t(\mathbb{Z})$, $\mathbb{Z}) \cong \mathbb{Z}$. Then $A$ is virtually cyclic and one direction of the proof is completed.

Now suppose that $A$ is virtually free, so $N_{A}(F) \cap C_{A}(Q)$ is virtually free. Of course, we may assume that $F$ is non-cyclic (else the desired conclusion is trivial) and so, $Z(F)=1$. It follows that if $\alpha \in C_{A u(F)}(\hat{G} / \hat{F})$, then for every $g \in G$, there is a unique element $f=f_{g}$ in $F$ such that $\alpha^{-1} \hat{g} \alpha=\hat{g} \hat{f}$. If $\beta: G \rightarrow G$ is defined by $g^{\beta}=g f_{g}$ it is routine to check that $\beta$ is an automorphism of $G$ and, in fact, $\beta \in N_{A}(F) \cap C_{A}(Q)$. From this, we see that $N_{A}(F) \cap C_{A}(Q) \cong C_{A u t(F)}(\hat{G} / \hat{F})$ and thus, $C_{A u t(F)}(\hat{G} / \hat{F})$ is virtually free. By [8, I.3.11], $C_{\text {Out }(F)}(\hat{G} / \hat{F})=C_{\text {Aut }(F)}(\hat{G} / \hat{F}) / \hat{F}$ is finite, as desired.

Loosely speaking, it seems that the finiteness of $C_{\text {out }(F)}(\chi(Q))$ reflects the intuitive idea that $\hat{G} / \hat{F}$ should be large enough to block the extension to $G$ of most non-inner automorphisms of $F$.

As mentioned earlier, Proposition 3.1 yields some more general examples of groups in the class $\mathscr{F}_{0}$. We cited the dihedral group $\mathbb{Z}_{2} * \mathbb{Z}_{2}$ as an obvious example of a group in $\mathscr{F}_{1}$. But in fact, by [6, Theorem 1], if $G$ is an amalgamated free product of any two finite groups, then so is $\operatorname{Aut}(G)$, whence $G \in \mathscr{F}_{1}$. We also cited finite-by-infinite cyclic groups as obvious examples of groups in $\mathscr{F}_{0} \backslash \mathscr{F}_{1}$. Such a group is a special type of HNN extension. Here again, it can be shown by a direct combinatorial argument that if $G$ is any $H N N$ extension $H_{K}^{*} t$ of a finite group $H$, then an automorphism of $G$ which centralizes $H$ must map $t$ to $a t^{ \pm 1} b$ for some $a, b \in H$. From this, it follows that $\operatorname{Out}(G)$ is finite and so any such group lies in $\mathscr{F}_{0} \backslash \mathscr{F}_{1}$.

Lemma 3.2. If $G / Z(G)$ is virtually cyclic, then $(G / Z(G))_{a b}$ is finite.

Proof. Let $N$ be a normal subgroup of $G$ containing $Z(G)$ such that $N / Z(G)$ is cyclic and $|G: N|=q$ is finite. $N$ is, of course, necessarily abelian. If $(G / Z(G))_{a b}$ is infinite, then $(G / Z(G))^{\prime}$ is finite and so, since $N / Z(G)$ is infinite, $N / Z(G)$ intersects $(G / Z(G))^{\prime}$ trivially. Therefore, $[G, N] \leqslant G^{\prime} \cap N \leqslant Z(G)$. If $x \in G$ and $y \in N$, it follows that $\left[x, y^{q}\right]=[x, y]^{q}=$ $\left[x^{q}, y\right]=1$. But then $N^{q} \leqslant Z(G)$, contradicting the fact that $N / Z(G)$ is infinite cyclic. 
The primary objective of this paper is:

Theorem 3.3. Let $G$ be any finitely generated group and let $r$ be the torsion-free rank of $Z(G)$. Then Aut $(G)$ is virtually free if and only if $Z(G)$ is finitely generated, $r \leqslant 2$ and $G / Z(G) \in \mathscr{F}_{r}$.

Proof. Let $A=A u t(G)$ and $Z=Z(G)$.

Suppose first that $A$ is virtually free. $G / Z$ is then also virtually free and so, by Proposition 2.3, $G$ contains a normal subgroup $F$ which is free of finite rank such that $Z \cap F=1$ and $|G: Z \times F|$ is finite (so $Z$ is finitely generated). Moreover, $D=C_{A}(Z) \cap$ $C_{A}(G / F)$ and $E=C_{A}(F) \cap C_{A}(G / Z)$ are isomorphic to subgroups of finite index in $A u t(G / Z)$ and $A u t(G / F)$ respectively. In particular, $A u t(G / Z)$ and $A u t(G / F)$ are each virtually free and $G / Z \in \mathscr{F}_{0}$ by Proposition 3.1.

If $D$ is finite, then $\operatorname{Aut}(G / Z)$ is finite and so $(G / Z) / Z(G / Z)$ is finite. By Schur's theorem $[12,10.1 .4],(G / Z)^{\prime}$ is finite. But by Theorem $2.1, G / Z$ is virtually cyclic and thus, Lemma 3.2 yields that $G / Z$ is finite (so it belongs to $\mathscr{F}_{2}$ ). To complete this case, it suffices to show that the torsion-free rank of $Z$ is at most two.

Let $W$ be a free abelian subgroup of finite index in $Z$. If $m=|G: W|$, then the transfer homomorphism $G \rightarrow W$ is just the power map $x \rightarrow x^{m}[12,10.1 .3]$. Let $P$ be the subgroup of $\operatorname{Aut}(W)$ consisting of all automorphisms which act trivially on $W / W^{m}$. If $\beta$ is any element of $P$, let $\eta$ be the endomorphism of $W$ defined by $\left(w^{\eta}\right)^{m}=[w, \beta]$ and define $\alpha$ : $G \rightarrow G$ by $x^{\alpha}=x x^{m \eta}$. ( $\eta$ is well-defined because $W$ is torsion-free.) Then because $x^{m} \in W \leqslant Z(G)$ for all $x \in G, \alpha$ is certainly an endomorphism of $G$ which induces the identity on $G / W$ and whose restriction to $W$ is $\beta$. It follows that $\alpha$ is an automorphism of $G$. (It is injective because, if $x \in k e r \alpha$, then $x=x^{-m n} \in W$ and so $x \in k e r \beta=1$. It is surjective because, if $y \in G$, then $y^{-1} y^{\alpha}=y^{m \eta} \in W$ and so $y^{-1} y^{\alpha}=w^{\beta}=w^{\alpha}$ for some $w \in W$. Thus, $y=\left(y w^{-1}\right)^{\alpha}$.) If $B$ is the subgroup of $A$ generated by all such $\alpha$, restriction defines an epimorphism $B \rightarrow P$ with kernel $C_{B}(W)$. But $C_{B}(W) \leqslant C_{A}(W) \cap C_{A}(G / W) \cong H o m(G / W, W)=$ 0 . Thus, $P \cong B$, whence $P$ is virtually free. But $|A u t(W): P| \leqslant\left|A u t\left(W / W^{m}\right)\right|$ which is finite and so, $A u t(W)$ is virtually free. Since $A u t(W) \cong G L_{r}(\mathbb{Z})$ where $r$ is the rank of $W\left(=\right.$ the torsion-free rank of $\mathbb{Z}$ ), and since $G L_{r}(\mathbb{Z})$ clearly contains a free abelian subgroup of rank $r-1$, it follows that $r \leqslant 2$. This completes the case that $D$ is finite.

Assume now that $D$ is infinite. Since $[D, E] \leqslant D \cap E \leqslant C_{A}(G / F) \cap C_{A}(G / Z)=1$ and $A$ is virtually free, $E$ must then be finite. Also, if $C=C_{A}(Z) \cap C_{A}(G / Z) \cong \operatorname{Hom}(G / Z, Z), C$ is a finitely generated abelian normal subgroup of $A$ and so $C$ has torsion-free rank at most one. Thus, $\operatorname{Aut}(C)$ is finite and so $D / C_{D}(C)$ is finite, whence $C_{D}(C)$ is infinite. Since $D \cap C \leqslant C_{A}(G / F) \cap C_{A}(G / Z)=1$, it follows that $C$ is finite (for otherwise, $C \times C_{D}(C)$ contains a rank two free abelian subgroup, contradicting the fact that $A$ is virtually free). So in this case, both $E$ and $C$ are finite.

Since $E$ is finite, $\operatorname{Aut}(G / F)$ is finite and so by Theorem $2.1, G / F$ is either finite or it is a finite central extension of an infinite cyclic group. Accordingly, $Z$ has torsion-free rank zero or one (since $Z \times F / F \leqslant Z(G / F)$ ). If the rank is zero, we are finished. If the rank is one, then since $\operatorname{Hom}(G / Z, Z) \cong C$ is finite, it follows that $(G / Z)_{a b}$ is finite, whence $G / Z$ belongs to $\mathscr{F}_{1}$. This completes one direction of the proof. 
Assume now that $Z$ is finitely generated with torsion-free rank $r$ at most two and $G / Z \in \mathscr{F}_{r}$. Since $G / Z \in \mathscr{F}_{0}, \operatorname{Aut}(G / Z)$ is virtually free by Proposition 3.1. We consider the following sections of $A$ :

$$
\begin{gathered}
A / C_{A}(G / Z) \hookrightarrow A u t(G / Z) \\
C_{A}(G / Z) / C_{A}(G / Z) \cap C_{A}(Z) \hookrightarrow A u t(Z), \text { and } \\
C_{A}(G / Z) \cap C_{A}(Z) \cong H o m\left((G / Z)_{a b}, Z\right) .
\end{gathered}
$$

If $r$ is at most one, $A u t(Z)$ is finite and since either $Z$ or $(G / Z)_{a b}$ is finite in this case, $\operatorname{Hom}\left((G / Z)_{a b}, Z\right)$ is finite. Since $\operatorname{Aut}(G / Z)$ is virtually free, so is $A$. If $r$ is two (so $G / Z$ is finite), then $A u t(G / Z)$ and $\operatorname{Hom}\left((G / Z)_{a b}, Z\right)$ are both finite and so, since $A u t(Z) \cong G L_{2}(Z)$ which is virtually free, $A$ is again virtually free. The proof of the theorem is now complete.

From a computational point of view, the difficulty with applying Theorem 3.3 is in verifying the centralizer condition that defines the class $\mathscr{F}_{0}$. Of course, for the subclass of virtually cyclic groups, this difficulty evaporates and so it is perhaps not surprising that we obtain a very simple characterization of groups whose automorphism groups have this structure.

Theorem 3.4. Let $G$ be a finitely generated group. Then $A u t(G)$ is virtually cyclic if and only if both $Z(G)$ and $G / Z(G)$ are virtually cyclic.

Proof. Suppose that $A=A u t(G)$ is virtually cyclic. Then certainly $G / Z(G) \cong \operatorname{Inn}(G)$ is virtually cyclic. However, the transfer argument in Theorem 3.3 (where it is shown that $r \leqslant 2$ ) shows that in this case, $r \leqslant 1$ (since $G L_{2}(\mathbb{Z}$ is not virtually cyclic). Thus, $Z(G)$ is virtually cyclic.

Assume now that both $Z=Z(G)$ and $G / Z$ are virtually cyclic. Then $\operatorname{Aut}(Z)$ is finite by Theorem 2.1 and so, $A / C_{A}(Z)$ is finite. Also, by Lemma $3.2,(G / Z)_{a b}$ is finite and so, $C_{A}(Z) \cap C_{A}(G / Z) \cong \operatorname{Hom}\left((G / Z)_{a b}, Z\right)$ is also finite. Finally, Proposition 3.1 implies that $\operatorname{Aut}(G / Z)$ is virtually free and in fact, the third paragraph of the proof of Proposition 3.1 shows that $\operatorname{Aut}(G / Z)$ is virtually cyclic. Thus, $A / C_{A}(G / Z)$ is virtually cyclic and it follows that $A$ is virtually cyclic.

Corollary 3.5. Let $G$ be a finitely generated group such that $A u t(G)$ is virtually cyclic. If $H$ is either a subgroup of finite index or a homomorphic image of $G$, then $\operatorname{Aut}(H)$ is also virtually cyclic.

A somewhat sharper conclusion about virtually cyclic automorphism groups can be gleaned from the following lemma:

Lemma 3.6. If $H$ is an infinite virtually cyclic group and $H_{a b}$ is finite, then $H$ is finite-by-dihedral. 
Proof. If $H$ satisies the hypotheses, it has a unique maximal finite normal subgroup $M$ and $H / M$ also satisfies the hypotheses. Thus, we may assume that $M$ is trivial. Let $N$ be an infinite cyclic normal subgroup of minimal finite index in $H$ and let $C=C_{H}(N)$ (so $|H: C| \leqslant|\operatorname{Aut}(N)|=2$ ). Then $N \leqslant Z(C)$, whence $C^{\prime}$ is finite by Schur's theorem [12, 10.1.4]. Since $H$ contains no non-trivial finite normal subgroups, it follows that $C$ is abelian and hence, because of the minimality of $|H: N|, C=N$. Since $H_{a b}$ is finite, $|H: N|=|H: C|=2$, and so if $x \in H, x \notin N$, then $H=\langle N, x\rangle$ and $x^{2} \in N$. It follows that $x^{2} \in Z(H)$. If $x^{2} \neq 1$, then $\left|N:\left\langle x^{2}\right\rangle\right|$ is finite, whence $H / Z(H)$ is finite. Again by Schur's theorem, $H^{\prime}$ is finite and so, $H_{a b}$ is infinite, contradicting the hypotheses. We conclude that $x^{2}=1$ and it follows that $H$ is dihedral.

Theorem 3.7. If $G$ is finitely generated and $A u t(G)$ is a finite extension of an infinite cyclic group, then both $\mathrm{Aut}(G)$ and $G / Z(G)$ are finite-by-dihedral.

Proof. By Theorem 3.4 , both $G / Z(G)$ and $Z(G)$ are virtually cyclic. Also, $G / Z(G)$ must be infinite, for otherwise, by Theorem 2.1, Aut $(G)$ is finite. From Lemmas 3.2 and 3.6, we conclude that $G / Z(G)$ is finite-by-dihedral. $(G / Z(G))^{\prime}$ is then infinite, whence $(\operatorname{Aut}(G))^{\prime}$ is certainly infinite. Therefore, $\operatorname{Aut}(G)_{a b}$ is finite and so, by Lemma 3.6, $A u t(G)$ is finite-by-dihedral. This completes the proof.

Corollary 3.8. A finite central extension of an infinite cyclic group cannot be the automorphism group of any finitely generated group.

In view of Corollary 3.8 (and perhaps also the observation that no free group is the automorphism group of any group [4]), one may ask about the possibility of infinite finite-by-free automorphism groups. The next result limits these possibilities but is followed by a construction for an infinite family of groups, each of whose automorphism groups is the direct product of a free and a finite group.

Theorem 3.9. If $G$ is finitely generated and $A u t(G)$ is an infinite finite-by-free group, then $G$ is a finite central extension of a free abelian group of rank two.

Proof. Of course, if $A u t(G)$ is finite-by-free then it is virtually free and so, by Theorem $3.3, Z(G)$ is finitely generated with torsion-free rank at most two. Suppose, in fact, that this rank is at most one. $G / Z(G) \cong \operatorname{Inn}(G)$ is finite-by-free and so $G / Z(G)$ contains a finite normal subgroup $H / Z(G)$ such that $G / H \cong(G / Z(G)) /(H / Z(G))$ is free. Thus, $G$ contains a free subgroup $F$ such that $G=F H$ and $F \cap H=1$. By Theorem 2.1, $\operatorname{Aut}(H)$ is finite and so, if $L=C_{F}(H)$, then $F / L$ is finite.

For any $\alpha$ in $C_{\text {Aut }(F)}(F / L)$, define a map $\beta: G \rightarrow G$ by $(f h)^{\beta}=f^{\alpha} h$ for all $f$ in $F$ and $h$ in $H$. It is routine to check that $\beta$ is an automorphism of $G$. This gives rise to a monomorphism from $C_{A u t(F)}(F / L)$ into $\operatorname{Aut}(G)$ and so $C_{A u t(F)}(F / L)$ is finite-by-free. But $\operatorname{Aut}(F) / C_{\text {Aut }(F)}(F / L)$ is finite (since $F / L$ is) and so, $\operatorname{Aut}(F)$ is virtually free. It is known that if $F_{2}$ is the free group of rank two, then $\operatorname{Aut}\left(F_{2}\right) / \operatorname{Inn}\left(F_{2}\right) \cong G L_{2}(\mathbb{Z})[8,1.4 .5]$ and so, $A u t\left(F_{2}\right)$ cannot be virtually free (by $[8,1.3 .11]$ ). Thus, we conclude that $F$ is cyclic, 
whence $L \leqslant Z(G)$. Since $Z(G) \leqslant H$ and $H \cap F=1$, it follows that $F=1$ and so $G=H$ and $\operatorname{Aut}(G)$ is finite, contradicting the hypotheses. We are forced to conclude that the torsion-free rank of $Z(G)$ is exactly two and thus, by Theorem $3.3, G / Z(G)$ is finite. The theorem follows.

Example. Let $m$ and $n$ be powers of $p$ and $q$ respectively where $p$ and $q$ are distinct primes for which g.c.d. $(p-1, q-1)>4$. If $u$ and $v$ are primitive roots modulo $m$ and $n$ respectively, then by the Chinese Remainder theorem, there exist integers $r$ and $s$ with $r \equiv u(\bmod m), r \equiv 1(\bmod m), s \equiv 1(\bmod n)$ and $s \equiv v(\bmod n)$. Let $G$ have the presentation

$$
\left\langle x, y, z: x^{m n}=1=[y, z], y^{-1} x y=x^{r}, z^{-1} x z=x^{s}\right\rangle .
$$

If $k=\phi(m), j=\phi(n)$ and $Z=\left\langle y^{k}, z^{j}\right\rangle$, then $Z$ is free abelian of rank two, $Z=Z(G)$, and $G / Z$ is isomorphic to the holomorph of the cyclic group of order $m n$. By a result of $\mathrm{G}$. A. Miller $[10], G / Z$ is complete and so, if $A=A u t(G)$, then $A=C \times \operatorname{Inn}(G)$, where $C=C_{A}(G / Z)$.

Suppose that $\alpha \in C$. Then $[y, \alpha]=y^{k a} z^{j c}$ and $[z, \alpha]=y^{k b} z^{j d}$ for certain integers $a, b, c$, and $d$. It follows that, with respect to the basis $\left\{y^{k}, z^{j}\right\}$, the restriction of $\alpha$ to $Z$ is represented by the matrix $\left(\begin{array}{cc}k a+1 & j c \\ k c & j d+1\end{array}\right)$. If $\alpha$ has finite order, the eigenvalues of this matrix are complex roots of unity and hence, its trace lies between -2 and 2 . Therefore, $4 \leqslant k a+j d \leqslant 0$. But g.c.d. $(k, j)=$ g.c.d. $(p-1, q-1)>4$, whence $k a+j d=0$ and the trace is 2 . Thus, both eigenvalues are 1 and $\alpha$ induces the identity map on $Z$. Since $C \cap C_{A}(Z) \cong$ $\operatorname{Hom}(G / Z, Z)=0, \alpha$ must be the identity. This proves that $C$ is torsion-free. However, $C \cong C / C_{A}(Z)$ is isomorphic to a subgroup of $A u t(Z) \cong G L_{2}(\mathbb{Z})$ and hence, $C$ is free. Thus, $\operatorname{Aut}(G)$ is the direct product of the finite group $\operatorname{Inn}(G)$ with a free group.

We conclude with two closure properties of the class $\mathscr{F}_{0}$, the first of which is almost immediate from Proposition 3.1.

Proposition 3.10. Let $G$ be a finitely generated virtually free group and suppose that $G \leqslant H$ with $|H: G|$ finite. If $\operatorname{Aut}(G)$ is virtually free, then Aut $(H)$ is virtually free. In other words, the class $\mathscr{F}_{0}$ is closed with respect to constructing supergroups of finite index.

Proof. From a glance at the proof of Proposition 3.1, it is evident that the virtual freeness of $A u t(G)$ implies the finiteness of $C_{O u(F)}(\hat{G} / \hat{F})$, regardless of which free normal subgroup $F$ of finite index in $G$ one chooses. Under the hypotheses above, we may choose $F$ to be normal in $H$ (since $|H: G|$ is finite). The finiteness of $C_{\text {out }(F)}(\hat{G} / \hat{F})$ certainly implies that $C_{\text {Out }(F)}(\hat{H} / \hat{F})$ is finite and so, $A u t(H)$ is virtually free.

Note. Without the hypothesis that $G$ is virtually free, the proposition above would be false. For example, if $G$ is free abelian of rank two, then $\operatorname{Aut}(G)=G L_{2}(\mathbb{Z})$ is virtually 
free. However, if $\alpha$ is the automorphism which inverts $G$ and $H$ is the semi-direct product $G\langle\alpha\rangle$, then $A u t(H)$ is not virtually free (since $\operatorname{Inn}(H) \cong H$ is not).

Corollary 3.11. If $G \in \mathscr{F}_{0}$ then $\operatorname{Aut}(G) \in \mathscr{F}_{0}$.

Proof. Let $G \in \mathscr{F}_{0}$. By Proposition 3.1, $|\operatorname{Aut}(G): \ln n(G)|$ is finite [8, I.3.11]. Thus, by Proposition 3.10, it suffices to show that $\operatorname{Inn}(G) \in \mathscr{F}_{0}$.

Let $F$ be a free normal subgroup of finite index in $G$. If $F$ is cyclic then $\operatorname{Aut}(\operatorname{Inn}(G))$ is virtually cyclic by Corollary 3.5 and so $\operatorname{In} n(G) \in \mathscr{F}_{0}$ by Proposition 3.1. Thus, we may assume that $F$ has rank at least two and in particular, $F \cap Z(G)=1$. If $\tilde{F}=F Z(G) / Z(G)$ and $\bar{G}=G / Z(G)$, let $\chi$ and $\bar{\chi}$ be the couplings corresponding to the obvious extensions $F \rightarrow G \rightarrow G / F$ and $\bar{F} \rightarrow \bar{G} \rightarrow \bar{G} / \bar{F}$ respectively. Then it is routine to check that $C_{\text {Out }(\bar{F})}(\bar{\chi}(\bar{G} / \bar{F})) \cong C_{\text {Out }(F)_{1}}(\chi(G / F))$ and hence, by Proposition 3.1, $\bar{G} \in \bar{F}_{0}$. Thus, $\operatorname{Inn}(G) \in \bar{F}_{0}$ and the result is proved.

In [6], it is remarked that if $G=\mathbb{Z} * \mathbb{Z}_{3}$, then $A=A u t(G)$ is the free product of two finite factors with an amalgamated subgroup (whence $A \in \mathscr{F}_{1}$ ) and moreover, in the automorphism sequence

$$
\operatorname{Aut}(A), \operatorname{Aut}(\operatorname{Aut}(A)), \ldots
$$

no two terms are isomorphic.

\section{REFERENCES}

1. J. L. Alperin, Groups with finitely many automorphisms, Pacific J. Math. 12 (1962), 1-5.

2. R. BAER, Finite extensions of abelian groups with minimum conditions, Trans. Amer. Math. Soc. 79 (1955), 521-540.

3. D. J. Collins, The automorphism group of a free product of finite groups, Arch. Math. (Basel) 50 (1988), 385-390.

4. J. L. DYER, A remark on automorphism groups, Contemp. Math 33 (1984), 208-211.

5. S. KalajDzIEvSKI, Automorphism group of a free group: centralizers and stabilizers, $J$. Algebra 150 (1992), 435-502.

6. A. Karrass, A. Pietrowski and D. Solitar, Automorphisms of a free product with an amalgamated subgroup, Contemp. Math. 33 328-340.

7. S. KRSTIC, Finitely generated virtually free groups have finitely presented automorphism groups, Proc. London Math. Soc. (3) 64 (1992), 49-69.

8. R. C. Lyndon and P. E. Schupp, Combinatorial Group Theory (Springer-Verlag, Berlin, 1977).

9. J. McCool, The automorphism groups of finite extensions of free groups, Bull. London Math. Soc. 20 (1988), 131-135. 
10. G. A. Miller, On the multiple holomorphs of a group, Math. Annalen 66 (1908), 133-142.

11. M. R. Pettet, The automorphism group of a virtual direct product of groups, in Proceedings of the A.M.S. Special Session, Infinite Groups and Group Rings, Tuscaloosa 1992 (World Scientific, Singapore, New Jersey, London, Hong Kong, 1993), 102-110.

12. D. J. S. Robinson, $A$ Course in the Theory of Groups (Springer-Verlag, New York, 1982).

13. J.-P. Serre, Trees (Springer-Verlag, Berlin, 1980).

Department of Mathematucs

UNIVERSITY OF TOLEDO

TOLEDO, OHı 43606

U.S.A. 